\title{
Coriocarcinoma gestacional primario de cuello uterino. Presentación de un caso*
}

\author{
(D) Viviana García, ${ }^{1}$ (D) Franco Calderaro Di Ruggiero, ${ }^{2}$ (D) Jorge Hoegl, ${ }^{3}$ \\ Carlos Quintero, ${ }^{4}$ (D) Johatson Freytez. ${ }^{1}$
}

\begin{abstract}
RESUMEN
El coriocarcinoma, representa un tipo de tumor maligno de la enfermedad trofoblástica gestacional. Puede desarrollarse después de un embarazo molar, un aborto espontáneo, un embarazo normal o ectópico. Generalmente su sitio de asiento es el cuerpo uterino, se han descrito lugares infrecuentes como el cérvix. Se reporta el caso de una paciente de 37 años, VI gestas IV partos I cesárea I embarazo molar, con sangrado uterino anormal, la cual es referida al Servicio Oncológico Hospitalario. Al examen ginecológico, se observa una masa exofítica en el cérvix. Se tomó biopsia que reportó: Coriocarcinoma gestacional y se comprobó niveles plasmáticos de $\beta$-hCG: 13805 UI/L. Se realizó histerectomía abdominal total con preservación de los ovarios. Se concluyó como estadio I de la Federación Internacional de Ginecología y Obstetricia y 8 según la puntuación de la Organización Mundial de la Salud (ST I: 8) de alto riesgo, por lo que se indicó adyuvancia. Actualmente sin evidencia de enfermedad.
\end{abstract}

Palabras clave: Coriocarcinoma, enfermedad trofoblástica gestacional, cuello uterino.

\section{SUMMARY}

Choriocarcinoma represents a type of malignant tumor of gestational trophoblastic disease. It can develop after a molar pregnancy, miscarriage, normal or ectopic pregnancy. Generally its seat site is the uterine body; infrequent places such as the cervix have been described. We report the case of a 37-year-old patient is reported, VI gestations IV deliveries I cesarean section I molar pregnancy, with abnormal uterine bleeding, which is referred to the Hospital Oncology Service. On gynecological examination, an exophytic mass is observed in the cervix. A biopsy was taken that reported: Gestational choriocarcinoma and plasma levels of $\beta$-hCG were verified: $13805 \mathrm{IU} / \mathrm{L}$. A total abdominal hysterectomy was performed with preservation of the ovaries. It was concluded as stage I of the International Federation of Gynecology and Obstetrics and 8, according to the score of the World Health Organization (ST I: 8), for which adjuvant was indicated. Currently no evidence of disease.

Keywords: Choriocarcinoma, gestational trophoblastic disease, cervix.

\section{INTRODUCCIÓN}

El coriocarcinoma representa un tipo de tumor maligno agresivo de la enfermedad trofoblástica gestacional.

\footnotetext{
${ }^{1}$ Residente del Postgrado de Ginecología Oncológica, UCV - SOH - IVSS. Caracas, Venezuela. ${ }^{2}$ Doctor en Ciencias de la Salud, Cirujano General - Oncólogo Ginecólogo Oncólogo. Jefe del Servicio y Director del Postgrado de Ginecología Oncológica, UCV - SOH - IVSS. Caracas, Venezuela. ${ }^{3}$ Ginecólogo Oncólogo, Adjunto del Servicio de Ginecología Oncológica, UCV - SOH - IVSS. Caracas, Venezuela. ${ }^{4}$ Cirujano General - Cirujano Oncólogo. Adjunto del Servicio de Ginecología Oncológica, UCV - SOH - IVSS. Caracas, Venezuela. *Premio al mejor Cartel de Ginecología, expuesto en el XXXIV Congreso de la Sociedad de Obstetricia y Ginecología de Venezuela / 2021). Correo de correspondencia: vivielisa25@gmail.com
}

Forma de citar este artículo: García V, Calderaro Di Ruggiero F, Hoegl J, Quintero C, Freytez J. Coriocarcinoma gestacional primario de cuello uterino. Presentación de un caso. Rev Obstet Ginecol Venez. 2021; 81(4):406-410. https://doi.org/10.51288/00810412
El riesgo estimado de desarrollarlo después de una mola hidatiforme completa es de $2 \%-3 \%$ y después de una mola parcial $<0,5 \%$, pudiendo aparecer en cualquier tipo de embarazo, $50 \%$ después de una mola hidatiforme, $25 \%$ proviene de abortos espontáneos, $22,5 \%$ de embarazos de más de 28 semanas y el 2,5\% de embarazos ectópicos (1). Generalmente su sitio de asiento es el cuerpo uterino, pero se describe aunque poco frecuente, en el cérvix (2).

En 1965, Saito y cols. (3) definieron los criterios diagnósticos del coriocarcinoma primario de cérvix.

A continuación, se presenta un caso de coriocarcinoma gestacional primario de cuello uterino. 


\section{CASO CLÍNICO}

Paciente de 37 años de edad, VI gestas, IV paras, I cesárea, I embarazo molar (a los 25 años), gestación a término sin complicaciones (a los 30 años), quien inició enfermedad actual de 9 meses de evolución, caracterizada por sangrado uterino anormal, tratado con antifibrinolíticos, sin mejoría. Fue evaluada por especialista, quien evidenció tumoración en cérvix, por lo que fue referida al Servicio de Ginecología Oncológica (SGO), del Servicio Oncológico Hospitalario (SOH), del Instituto Venezolano de los Seguros Sociales (IVSS). Al examen ginecológico, se evidenció el cuello uterino con pérdida de la arquitectura, dado por una masa exofítica, nodular, friable, de aproximadamente $6 \mathrm{~cm}$, que no comprometía el 1/3 superior de vagina ni los parametrios. Se tomó biopsia de la lesión, que reportó coriocarcinoma gestacional. Se constataron niveles de subunidad beta de gonadotrofina coriónica ( $\beta$-hCG): 13805 UI/L. Se realizó ultrasonido pélvico y transvaginal, demostrando ausencia de embarazo intrauterino o enfermedad en cuerpo uterino. En la radiografía de tórax no se evidenciaron lesiones en campos pulmonares, al igual que en la tomografía axial computarizada (TAC) de abdomen y pelvis con doble contraste, que no evidenciaron lesiones.

Se discutió el caso en la reunión del Servicio de Ginecología Oncológica y se planteó tratamiento quirúrgico. Fue llevada a mesa operatoria donde se realizó histerectomía abdominal total con conservación de ovarios.

En el estudio anatomopatológico se describió: útero de $9,5 \times 5 \times 3,5 \mathrm{~cm}$, con lesión confinada al cuello uterino, friable, violácea, nodular, de $8 \times 4 \mathrm{~cm}$, con márgenes negativos en el espécimen estudiado (Figuras 1 y 2).

En el reporte histológico, se describieron dos componentes celulares, el primero corresponde al

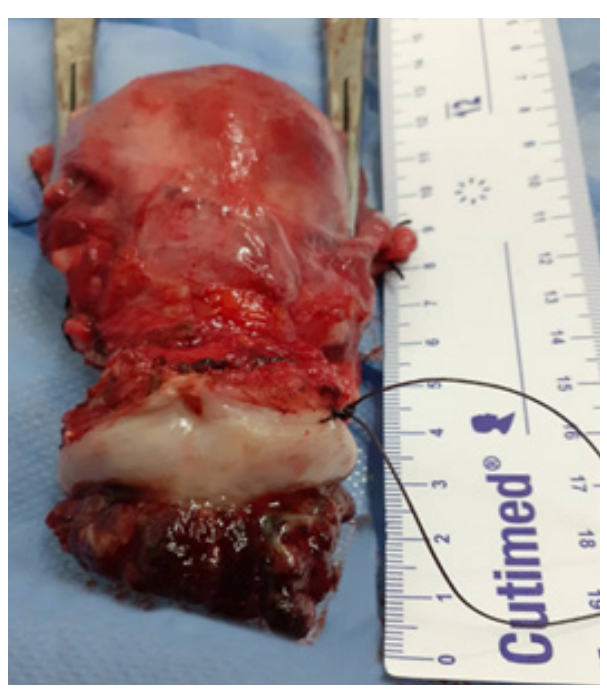

Figura 1. Espécimen quirúrgico: útero con lesión en cérvix.

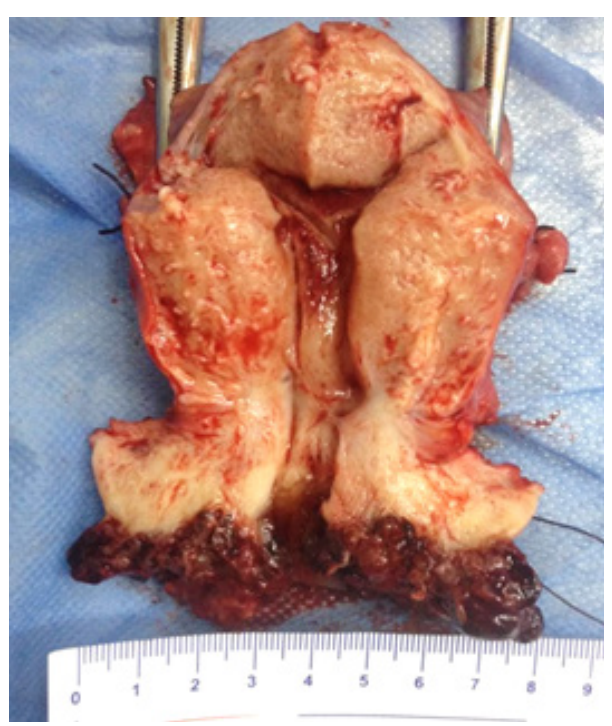

Figura 2. Corte longitudinal de la pieza quirúrgica, que muestra lesión exofítica, friable, confinada al cérvix.

citotrofoblasto, compuesto por células mononucleadas de tamaño pequeño o mediano, con escaso citoplasma claro, bordes nítidos citoplasmáticos, y el segundo representado por el sincitiotrofoblasto de aspecto multinucleado, con escasa actividad mitótica y 


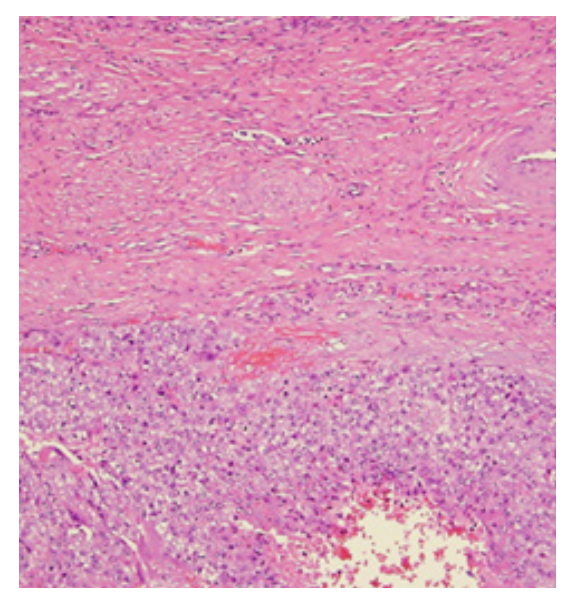

Figura 3. Corte histológico del cérvix: se muestra crecimiento bifásico, áreas de citotrofoblasto y sincitiotrofoblasto. (HE 200X).

amplio citoplasma eosinofílico, observándose en este, espacios vasculares que forman el sincitiotrofoblasto (Figura 3).

Se estadificó acorde a la Federación Internacional de Ginecología y Obstetricia (FIGO) como coriocarcinoma gestacional primario de cuello uterino, estadio (ST) I y score de riesgo de la Organización Mundial de la Salud (OMS) 8: edad menor de 40 años, gestación molar previa, con intervalo entre esta y aquella, de más de 12 meses, valores de $\beta$-hCG y el tamaño tumoral (ST I: 8) de alto riesgo, por lo que se indicó adyuvancia con esquema EMA/CO (etopósido, metotrexato, actinomicina $\mathrm{D}$, ciclofosfamida, vincristina) cada 21 días por 6 ciclos. La paciente toleró adecuadamente este esquema de quimioterapia, observándose descenso progresivo de $\beta$-hCG: 138,23 UI/L (después del ciclo $3 / 6)$ y $0,11 \mathrm{UI} / \mathrm{L}$ posterior al ciclo $6 / 6$. Actualmente permanece con controles séricos del biomarcador y sin evidencia de enfermedad.

\section{DISCUSIÓN}

El coriocarcinoma cervical primario representa una forma de coriocarcinoma ectópico. Su presentación es rara y con pocos casos documentados. La primera descripción de esta entidad data de 1915, por Alfieri y cols. (4). La revisión más extensa del coriocarcinoma ectópico fue hecha en Japón, por Saito y cols. (3), en 1965, quienes lo definieron como solo aquellos casos de coriocarcinoma sin afectación del cuerpo uterino.

El coriocarcinoma cervical primario por lo tanto, es un coriocarcinoma ectópico que surge en el cuello uterino. En esa revisión de 243 casos reportados como coriocarcinoma ectópico en la literatura japonesa, entre 1926 y 1963, se encontraron 119 casos que cumplieron los criterios. Las ubicaciones más comunes fueron pulmón (30 casos) y ovario (22 casos). El coriocarcinoma cervical representó solo el 15,1\% (18 casos) de estos (3).

Los criterios para el diagnóstico descritos por este autor se citan a continuación (Saito y cols. (3), 1965):

1. No existencia de foco primario en el cuerpo uterino.

2. Diagnóstico histopatológico confirmado de coriocarcinoma.

3. No existencia de mola hidatiforme o embarazo intrauterino normal.

4. El coriocarcinoma intramural de cuerpo uterino no debe considerarse como carcinoma ectópico.

Se desconocen los factores que determinan la etiopatogenia de esta entidad; sin embargo, se han postulado algunas hipótesis al respecto (5): a) la posibilidad de un embarazo cervical previo, que sufriría una transformación maligna posteriormente (6); b) se ha sugerido que el coriocarcinoma cervical, podría corresponder a una metástasis de un tumor primario localizado en el cuerpo uterino, que sufriría una regresión completa (7); y, por último, c) se ha propuesto el origen en el implante cervical 


\section{CORIOCARCINOMA GESTACIONAL PRIMARIO DE CUELLO UTERINO.}

PRESENTACIÓN DE UN CASO

de células coriónicas procedentes de un embarazo previo, que permanecerían latentes durante un tiempo indeterminado hasta sufrir una transformación maligna (8).

El diagnóstico de coriocarcinoma primario cervical, generalmente es difícil. Muchas veces se realiza por evaluación del espécimen posquirúrgico (9). El síntoma más frecuente es sangrado vaginal anormal, que en ocasiones puede ser una hemorragia masiva (10). En este caso, la paciente consultó por sangrado y se evidenció un tumor que ocupaba el cuello uterino. El diagnóstico se confirmó mediante el estudio histológico, en donde se observó un crecimiento celular bifásico con unas células citotrofoblásticas mononucleadas con citoplasma amplio y otras correspondientes a sincitiotrofoblasto, multinucleadas, gigantes, con amplios citoplasmas eosinófilos y grandes vacuolas (11). La determinación de la $\beta$-hCG, es importante para la confirmación del diagnóstico y valorar el pronóstico y el seguimiento (12). La evaluación por ecografía, permite descartar la presencia de embarazo intrauterino, o enfermedad en la cavidad uterina (13), tal como se realizó en este caso. El uso de doppler color puede ser útil en el diagnóstico, porque identifica el coriocarcinoma cervical como una lesión hipervascularizada (14).

Se describe que la incidencia de metástasis es baja, y con mejor pronóstico que otros coriocarcinomas ectópicos (15). Las metástasis generalmente ocurren en el cerebro y el hígado en $20 \%$ a $60 \%$ de los casos $\mathrm{y}$, ocasionalmente, en los ganglios linfáticos. Por esto, la evaluación sugerida debe incluir estudios de imagen de cerebro, tórax, abdomen y pelvis (10).

El tratamiento de elección sugerido, al igual que en la paciente cuyo caso que se presenta, es la histerectomía abdominal total seguida de quimioterapia (16).
Para el diagnóstico diferencial, debe distinguirse de un carcinoma indiferenciado y variantes de tumores epitelioides del músculo liso. El diagnóstico preciso se facilita mediante inmunohistoquímica, porque las células del coriocarcinoma cervical, expresan marcadores trofoblásticos variables como hCG, inhibina, antígeno leucocitario humano-G y Mel-CAM (CD146) (17). En casos de la localización extrauterina del tumor, es necesario diferenciar si tiene un origen gestacional o no gestacional, esto se logra mediante genotipificación molecular, demostrando positividad inmunohistoquímica para p57KIP2 (18). En este caso no hubo necesidad de recurrir a estos métodos.

\section{CONCLUSIÓN}

- El coriocarcinoma primario de cuello uterino es una entidad muy poco frecuente

- El apoyo para el diagnóstico, se basa en los valores de $\beta$-hCG y en la evaluación con estudios de extensión por imagen de manera oportuna para la detección de metástasis, y así alcanzar una correlación detallada de estos, para evitar errores diagnósticos y ofrecer un tratamiento adecuado

- Los casos reportados sugieren buenos resultados al tratamiento quirúrgico, seguido de quimioterapia

- Es necesario el control de la respuesta con el marcador sérico, como ocurrió en el presente caso

\section{REFERENCIAS}

1. Lurain JR. Gestational trophoblastic disease I: epidemiology, pathology, clinical presentation and diagnosis of gestational trophoblastic disease, and management of hydatidiform mole. Am J Obstet Gynecol. 2010; 203(6):531-539. doi: 10.1016/j. ajog.2010.06.073. 
2. Herts BR, Yee JM, Porges RF. Primary cervical choriocarcinoma: case report and review of the literature. J Ultrasound Med. 1993; 12(1):59-62. doi: 10.7863/jum.1993.12.1.59.

3. Saito M, Azuma T, Nakamura K. On ectopic choriocarcinoma. World Obstet Gynecol. 1965; 17:459484.

4. Alfieri E. Contributo allo studio del corioepitelioma cervicale con pungo periodo di latenza. Folia Gynaec. 1915; 10:371.

5. Tsukamoto N, Nakamura M, Kashimura M, Saito T. Primary cervical choriocarcinoma. Gynecol Oncol. 1980; 9(1):99-107. doi: 10.1016/0090-8258(80)90014-1.

6. Rashbaum M, Daub WW, Lisa JR. Primary cervical choriocarcinoma. Am J Obstet Gynecol. 1952; 64(2):451-5. doi: 10.1016/0002-9378(52)90330-x.

7. Novak E, Koff AK. Chorioepithelioma, with especial reference to disappearance of the primary uterine tumor. Am J Obstet Gynecol. 1930; 20:153164.

8. Acosta-Sison H. Apparent metastatic chorioepithelioma without demonstrable primary chorionic malignancy in the uterus: report of 3 cases; a new possible explanation of its occurrence. Obstet Gynecol. 1957; 10(2):165168.

9. Lee JD, Chang TC, Lai YM, Hsueh S, Soong YK. Choriocarcinoma of the cervix. Acta Obstet Gynecol Scand. 1992; 71(6):479-481. doi: 10.3109/00016349209021099.

10. Martin BR 3rd, Orr JW Jr, Austin JM Jr. Cervical choriocarcinoma associated with an intrauterine contraceptive device: a case report. Am J Obstet Gynecol. 1983; 147(3):343-344. doi: 10.1016/00029378(83)91127-4.

11. Salado M, López-Cepero JM, Soldevilla S, Merino $\mathrm{JM}$, Diéguez JA, Rodríguez-Fernández $\mathrm{A}$, et al. Coriocarcinoma primario de cérvix. Clin Invest Ginecol Obstet. 2001; 28(1):38-40. doi: 10.1016/ S0210-573X(01)77059-1
12. Philippe E, Dreyfus M. Maladies trophoblastiques gestationnelles. En: Encycl Med Chir Obstétrique. París: Elsevier; 1998. p. 12-27.

13. Ben-Chetrit A, Yagel S, Ariel I, Zacut D, Shimonovitz S, Celnikier-Hochner D. Successful conservative management of primary nonmetastatic cervical choriocarcinoma. Am J Obstet Gynecol. 1990; 163(4 Pt 1):1161-3. doi: 10.1016/0002-9378(90)90679-2.

14. Yahata T, Kodama S, Kase H, Sekizuka N, Kurabayashi $\mathrm{T}$, Aoki Y, et al. Primary choriocarcinoma of the uterine cervix: clinical, MRI, and color Doppler ultrasonographic study. Gynecol Oncol. 1997; 64(2):274-278. doi: 10.1006/gyno.1996.4541.

15. Daiko Z. [Clinical study of 120 cases of chorioepithelioma and destructive hydatidiform mole-their characteristic difference]. Nihon Sanka Fujinka Gakkai Zasshi. 1966; 18(9):1083-1092. Japanese.

16. Behtash N, Zarchi M, Shamshirsaz A, Shamshirsaz A, Miratashi-Yazd A, Mehrdad N. Primary Cervical Choriocarcinoma: Case Report and the Review of Literatures, J Ca Ther. 2013; 4(8):1335-1340. doi: 10.4236/jct.2013.48158.

17. Kalhor N, Ramirez PT, Deavers MT, Malpica A, Silva EG. Immunohistochemical studies of trophoblastic tumors. Am J Surg Pathol. 2009; 33(4):633-638. doi: 10.1097/PAS.0b013e318191f2eb.

18. Zhao J, Xiang Y, Wan XR, Feng FZ, Cui QC, Yang $\mathrm{XY}$. Molecular genetic analyses of choriocarcinoma. Placenta. 2009; 30(9):816-820. doi: 10.1016/j. placenta.2009.06.011. 